\title{
Iatrogenic Microperforation Linked to Hemorrhagic Pericardial Effusion Complicated by Cardiac Tamponade
}

\author{
Arun Philip ${ }^{1}$ \\ 1. Internal Medicine, Methodist Health System, Dallas, USA
}

Corresponding author: Arun Philip, arunphilip@mhd.com

\begin{abstract}
Pericardial effusions have a wide range of etiologies, including infection, inflammation, and malignancy. A complication of pericardial effusion is cardiac tamponade. In instances of cardiac tamponade, prompt echocardiography and stabilization are paramount in preventing mortality. Here, we report a case of iatrogenic microperforation of the right ventricle during a pacemaker lead adjustment causing a delayed pericardial effusion complicated by cardiac tamponade. Lead removal is recommended in cases of valvular endocarditis, pocket infection, thrombosis, or life-threatening dysrhythmias; however, there are no established guidelines in the setting of perforation. In this case, an emergent pericardiocentesis was performed due to cardiac tamponade, but lead extraction was not performed.
\end{abstract}

Categories: Cardiology, Internal Medicine

Keywords: cardiac tamponade, pericardial effusion, pacemaker lead perforation

\section{Introduction}

The pericardium is a sac surrounding the heart that contains a thin layer of fluid. A pericardial effusion occurs when there is an abnormally large amount of fluid in this layer. Pericardial effusions have a wide range of etiologies, including infection, inflammation, and malignancy [1]. Effusions can be acute or chronic, and the pericardium can expand with increases in fluid. They are challenging to identify as they can present asymptomatically to sudden cardiac death.

One of the most severe complications of a pericardial effusion is cardiac tamponade. Hemorrhagic pericardial effusions complicated by cardiac tamponade are typically caused by malignancy, percutaneous interventional procedures, post-pericardiotomy syndrome, and complications of myocardial infarctions [2]. Cardiac tamponade occurs when intrapericardial pressure increases to compress all cardiac chambers and impede cardiac filling. As tamponade progresses, intracardiac chamber volumes and diastolic compliance decreases resulting in decreased cardiac output. With further progression of cardiac tamponade, patients can suffer shock and death.

Received 06/10/2020

Review began 06/24/2020

Review ended 06/28/2020

Published 07/06/2020

(c) Copyright 2020

Philip. This is an open access article distributed under the terms of the Creative Commons Attribution License CC-BY 4.0., which permits unrestricted use, distribution, and reproduction in any medium, provided the original author and source are credited.
Perforations following pacemaker implantation can be defined as acute/early ( $<24$ hours after implantation), subacute (up to one month), or chronic. Multiple case reports of early ( $<24$ hours after implantation) iatrogenic right ventricle perforation after device implantation have been reported; however, this presentation is usually accompanied by chest pain and shortness of breath [3,4]. We present an uncommon cause of hemorrhagic pericardial effusion complicated by cardiac tamponade in which iatrogenic microperforation of the right ventricle occurred during a lead adjustment.

\section{Case Presentation}

A 77-year-old male presented to the emergency room with a two-week history of abdominal pain and dyspnea on exertion. The patient had a history of complete heart block with permanent pacemaker placement (two years prior to admission) and a recent diagnosis of nonvalvular atrial fibrillation on apixaban. Physical exam revealed that the patient was tachycardic. A complete blood count, electrolyte panel, and serum creatinine were unremarkable. The initial troponin value was elevated $(0.033 \mathrm{mg} / \mathrm{mL})$, and electrocardiogram showed sinus tachycardia (Figure 1). 


\section{Cureus}

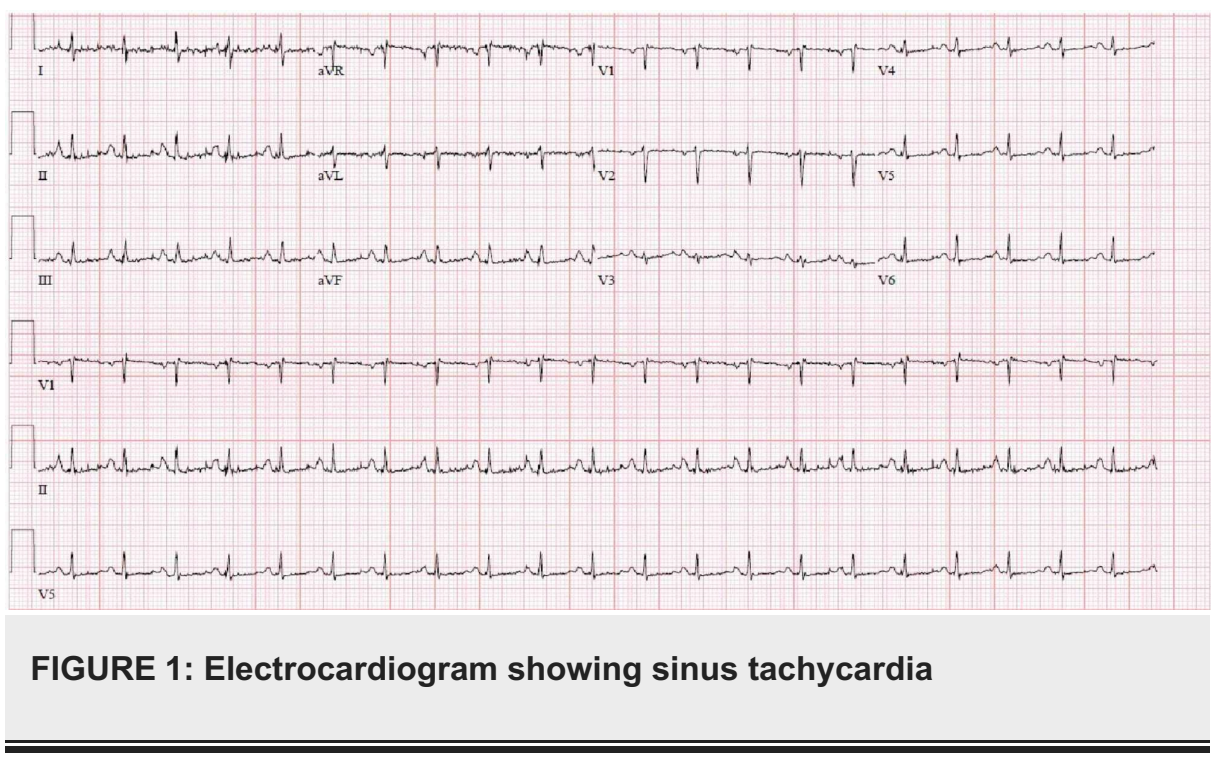

CT of the chest revealed a pericardial effusion (Figure 2).

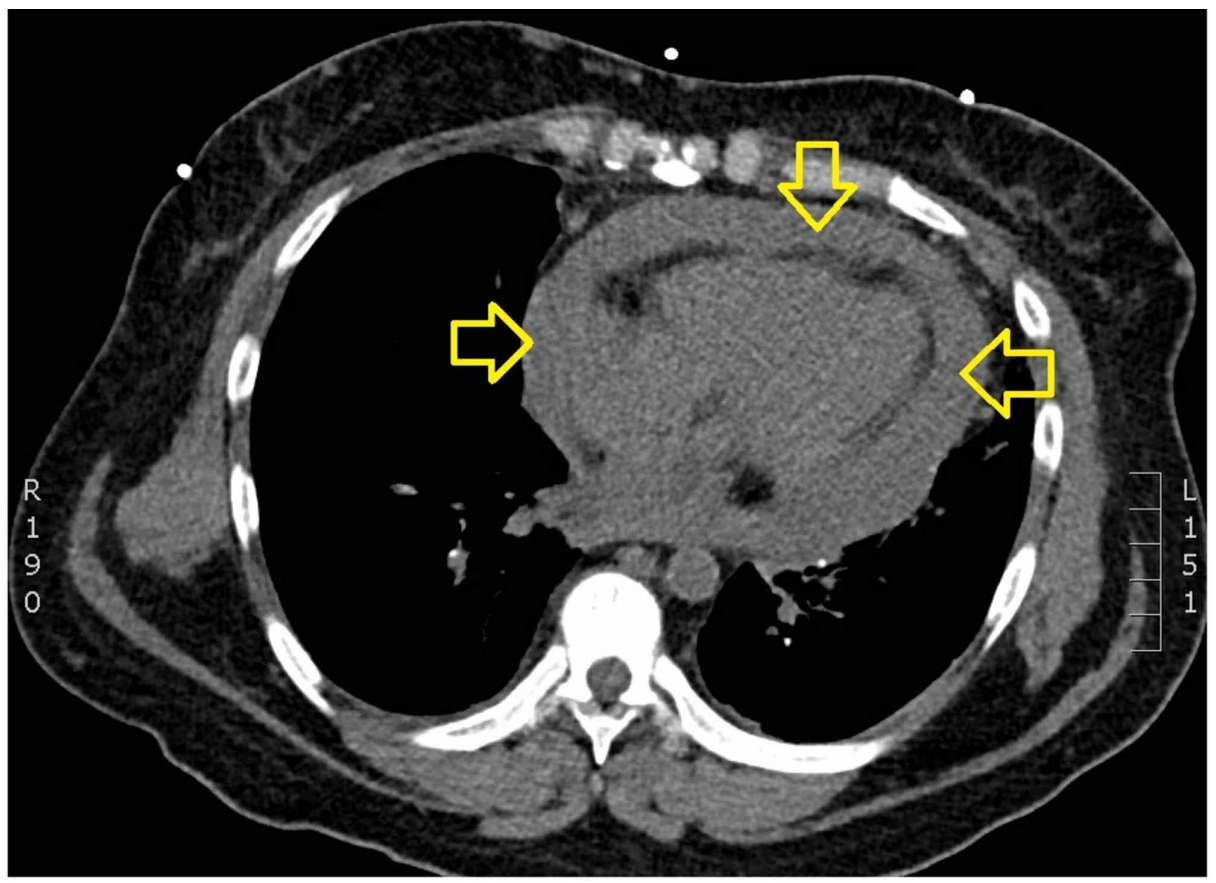

FIGURE 2: CT showing large pericardial effusion

A transthoracic echocardiogram (TTE) showed circumferential pericardial effusion with findings consistent with cardiac tamponade (Figure 3). 


\section{Cureus}

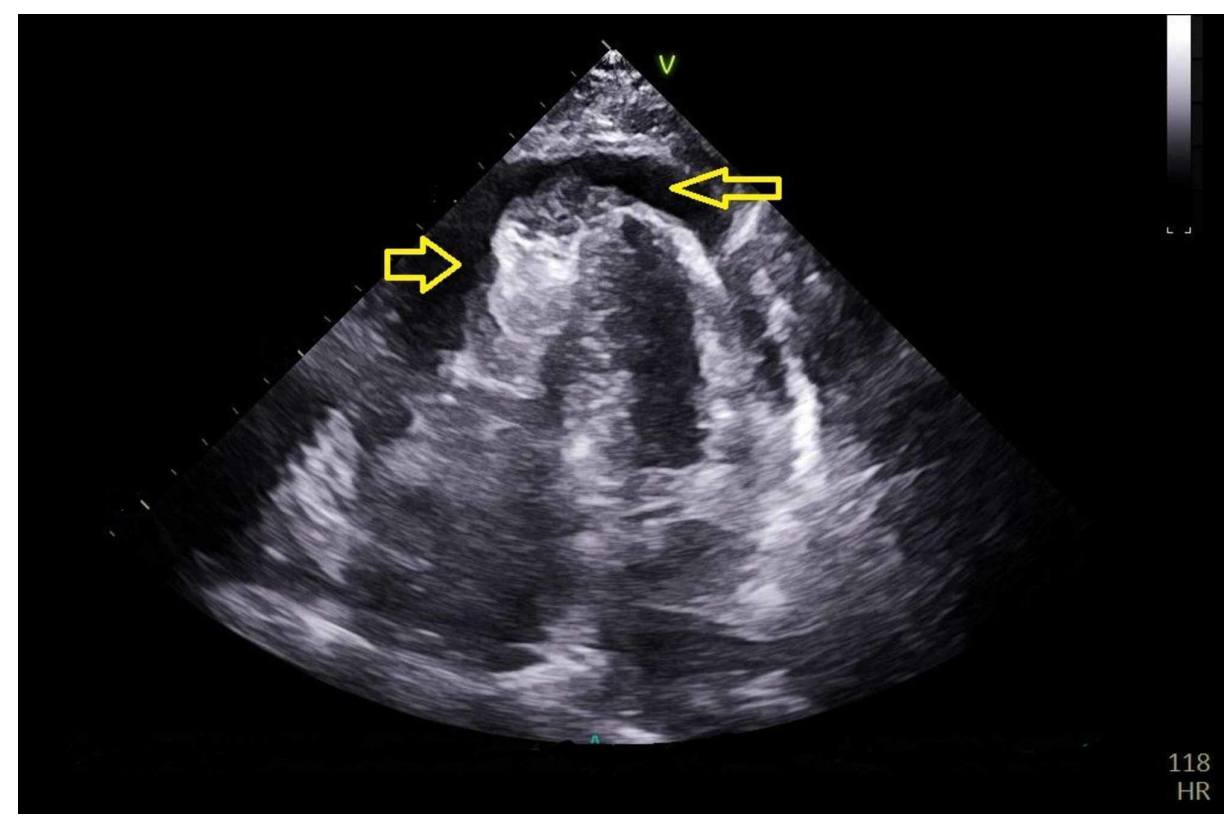

FIGURE 3: Transthoracic echocardiogram showing pericardial effusion

Cardiology was consulted and emergent pericardiocentesis was performed, in which $560 \mathrm{~mL}$ of hemorrhagic fluid was drained from the effusion (Figure 4).

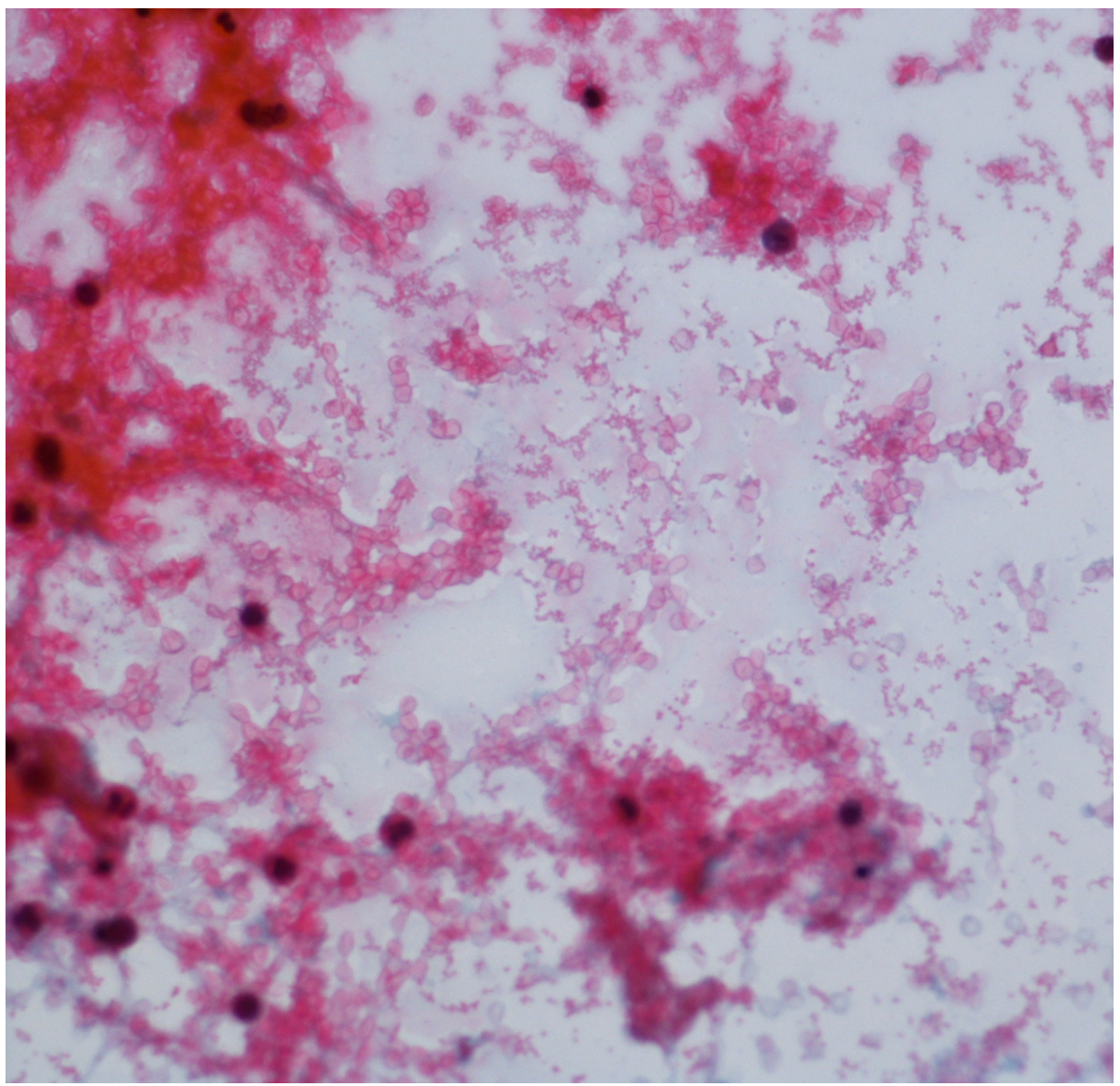

FIGURE 4: Fluid analysis of pericardial fluid shows abundant red blood cells with acute inflammatory cells with rare benign mesothelial cells. No malignant cells were identified. 
A repeat TTE showed no pericardial effusion after drainage. Gram, fungal, and mycobacterial stains were negative, and bacterial, fungal, and mycobacterial cultures of the pericardial fluid showed no growth. Cytologic evaluation showed no evidence for malignancy and serum thyroid-stimulating hormone was normal. Together, these results ruled out hypothyroidism, malignancy, and infectious etiologies for the pericardial effusion. The patient's records showed that his cardiologist had adjusted the patient's pacemaker leads two weeks prior to admission. It was determined that the pericardial effusion was most likely iatrogenic due to microperforation of the right ventricle during pacemaker lead adjustment, with bleeding exacerbated by apixaban use. The patient's abdominal pain resolved, and he was discharged home with outpatient cardiology follow-up.

\section{Discussion}

Myocardial perforation is a possible complication of permanent pacemaker implantation. Iatrogenic causations (e.g., transcatheter intervention and pacemaker insertion) have been implicated as causes of hemorrhagic pericardial effusions presenting with cardiac tamponade [1-3]. Other causes of pericardial effusions include malignancy (breast, lung, and lymphoma), complications from acute myocardial infarction, and uremia [3].

Right ventricular perforation is an uncommon cause of pericardial effusion, occurring in $0.1 \%$ to $0.8 \%$ of pacemaker placements [4-6]. Risk factors for ventricular perforation include the use of active fixation leads compared to passive fixation leads, low volume operators (less than 50 annual cases), female gender, steroid use, anticoagulant use, age greater than 75 years, body mass index (BMI) less than 25, chronic lung disease, and history of coronary intervention [7]. Symptoms of ventricular perforation can range from asymptomatic to life-threatening and include hiccups, chest pain, syncope, abdominal pain, and dyspnea [4].

Signs of right ventricle perforation include mammary hematoma, significantly decreased right ventricular pacing impedance, loss of ventricular capture (failure of generator stimulus to depolarize atrium or ventricle), and increased pacing threshold (observed on pacemaker interrogation) [4-6]. Chest x-rays can show migrated leads out of the heart silhouette as well as pleural or pericardial effusion, while TTE can show tips of leads in the pericardial space [6-8]. Fluoroscopy can be used to demonstrate abnormal motion or a hinge point of a lead trapped in the myocardium. CT can be helpful if other modalities are nondiagnostic; however, there can be difficulty in precisely locating the lead tip due to artifact $[7,8]$.

In the current case, an emergent pericardiocentesis was performed due to tamponade; however, lead extraction was not deemed necessary during this admission. The necessity of lead extraction, and the optimal means of extraction, whether percutaneous or surgical, remains a matter of controversy [4]. Lead extraction is necessary in patients with life-threatening dysrhythmias, when the lead interferes with the operation of implanted cardiac devices, and if the lead interferes with treatment of a malignancy $[9,10]$. Many cases can be managed by lead repositioning; however, increased length of time since implantation is associated with an increased risk of fibrotic adhesions. Recent guidelines suggest if the lead tip is inside the mediastinum and there is no active bleeding, an additional lead can be placed without performing lead extraction. However, the potential for further migration needs to be evaluated. If uncontrolled bleeding occurs or if the lead migrates outside the pericardium, then lead extraction must be performed [9]. Cardiotomy with surgical removal is typically reserved for cases in which transcutaneous approaches have failed, for hemodynamically unstable patients, or for patients with respiratory compromise [8-10].

\section{Conclusions}

Cardiac perforation is an extremely rare etiology of pericardial effusion; however, it is associated with significant morbidity and mortality. It should be suspected in patients presenting with a history of recent cardiac intervention and hemorrhagic pericardial effusion.

\section{Additional Information \\ Disclosures}

Human subjects: Consent was obtained by all participants in this study. Conflicts of interest: In compliance with the ICMJE uniform disclosure form, all authors declare the following: Payment/services info: All authors have declared that no financial support was received from any organization for the submitted work. Financial relationships: All authors have declared that they have no financial relationships at present or within the previous three years with any organizations that might have an interest in the submitted work. Other relationships: All authors have declared that there are no other relationships or activities that could appear to have influenced the submitted work.

\section{Acknowledgements}

We acknowledge Dr. Anne Murray for helping in writing the article, Dr. Neena Philip for locating the case, and Catherine Schack for assisting me in background research. 


\section{Cureus}

\section{References}

1. Corey GR, Campbell PT, Van Trigt P, et al.: Etiology of large pericardial effusions. Am J Med. 1993, 95:209213. 10.1016/0002-9343(93)90262-n

2. Atar S, Chiu J, Forrester JS, Siegel RJ: Bloody pericardial effusion in patients with cardiac tamponade: is the cause cancerous, tuberculous, or iatrogenic in the 1990s?. Chest. 1999, 116:1564-1569. 10.1378/chest.116.6.1564

3. Kiviniemi MS, Pirnes MA, Eränen HJK, Kettunen RVI, Hartikainen JEK: Complications related to permanent pacemaker therapy. Pacing Clin Electrophysiol. 1999, 22:711-720. 10.1111/j.1540-8159.1999.tb00534.x

4. Migliore F, Zorzi A, Bertaglia E, et al.: Incidence, management, and prevention of right ventricular perforation by pacemaker and implantable cardioverter defibrillator leads. Pacing Clin Electrophysiol. 2014, 37:1602-1609. 10.1111/pace.12472

5. Laborderie J, Barandon L, Ploux S, et al.: Management of subacute and delayed right ventricular perforation with a pacing or an implantable cardioverter-defibrillator lead. Am J Cardiol. 2008, 102:1352-1355. 10.1016/i.amicard.2008.07.025

6. Refaat MM, Hashash JG, Shalaby AA: Late perforation by cardiac implantable electronic device leads: clinical presentation, diagnostic clues, and management. Clin Cardiol. 2010, 33:466-475. 10.1002/clc.20803

7. Vamos M, Erath JW, Benz AP, Bari Z, Duray GZ, Hohnloser SH: Incidence of cardiac perforation with conventional and with leadless pacemaker systems: a systematic review and meta-analysis. J Cardiovasc Electrophsiol. 2017, 28:336-346. 10.1111/jce.13140

8. Khalid M, Murtaza G, Ayub MT, Ramu V, Paul T: Right ventricle perforation post pacemaker insertion complicated with cardiac tamponade. Cureus. 2018, 10:e2266. 10.7759/cureus.2266

9. Wilkoff BL, Love CJ, Byrd CL, et al.: Transvenous lead extraction: Heart Rhythm Society expert consensus on facilities, training, indications, and patient management: this document was endorsed by the American Heart Association (AHA). Heart Rhythm. 2009, 6:1085-1104. 10.1016/j.hrthm.2009.05.020

10. Kusumoto FM, Schoenfeld MH, Wilkoff BL, et al.: 2017 HRS expert consensus statement on cardiovascular implantable electronic device lead management and extraction. Heart Rhythm. 2017, 14:503-551. 10.1016/j.hrthm.2017.09.001 